\title{
Speed of Information Processing and Working Memory in Children with Cochlear Implants
}

\author{
Reid Herran ${ }^{1}$, David Pisoni ${ }^{1}$, William Kronenberger ${ }^{1,2}$ \\ ${ }^{1}$ Indiana University School of Medicine, DeVault Otologic Research Laboratory, Department of \\ Otolaryngology-Head \& Neck Surgery, ${ }^{2}$ Indiana University School of Medicine, Department of \\ Psychiatry
}

Background: Cochlear implants (Cls) restore partial hearing to deaf children, promoting the development of spoken language skills. However, because of reduced auditory and language experience, children who receive Cls are at risk for delays not only in language skills but also in language-related neurocognitive skills such as verbal working memory (VWM - the ability to retain language information in immediate memory concurrently with other cognitive processing). Although VWM delays in children with Cls are well-documented, the foundational processes underlying these delays are less clear. This study investigated the hypotheses that slower speed of information processing during VWM tasks contributes to VWM delays in $\mathrm{Cl}$ users and that this slower information processing speed is associated with spoken language outcomes.

Methods: 25 early-implanted, prelingually-deaf children with Cls and 25 normal-hearing $(\mathrm{NH})$ peers completed tests of VWM, neurocognitive, and speech-language functioning. Speed of information processing during the VWM test was assessed by measuring response latency and average pause duration.

Results: Children with Cls showed poorer VWM scores than NH peers, but the groups did not differ on response latencies or pause durations. Response latencies were significantly correlated with WWM capacity, speech, and language outcomes in both groups.

Conclusion: Speed of information processing in VWM was similar for children with Cls and $\mathrm{NH}$. In both groups, shorter response latencies (faster speed of execution of the cognitive operations of working memory) were associated with better neurocognitive and spoken language outcomes. In the $\mathrm{Cl}$ sample, pause durations were inconsistently associated with VWM and language outcomes.

Clinical Policy Impact and Implications: Speed of information processing for VWM is associated with core neurocognitive and spoken language outcomes for children with $\mathrm{Cls}$ and should be a routine target of assessment and intervention post-implantation. 